\title{
Projection of holographic images in volumetric fluorescent fluids for near-eye displays
}

\author{
Michał Makowski, Paweł Piątek, Mateusz Grynkiewicz
}

Faculty of Physics, Warsaw University of Technology, 75 Koszykowa, 00-662 Warsaw, Poland

Received September 26, 2019; accepted December 23, 2019; published December 31, 2019

\begin{abstract}
An optical setup is presented for holographic projection on scatterings in fluorescent liquids. Such media can be used as volumetric screens for near-eye holographic displays, solving the problem of speckle noise and very small exit pupils in existing setups. Three different oils (canola, olive and engine oil) are used with a $532 \mathrm{~nm}$ laser and tonic water with a $405 \mathrm{~nm}$ laser for projecting holographic fields, and the quality of such images is investigated. The laser wavelength is cut out from the acquisition on a camera and only filtered fluorescent light is observed. The best and brightest results are obtained with engine oil
\end{abstract}

Holographic technology allows users to see the 3D image of an object as if it was actually there. State of the art holographic displays can produce these images almost in real-time [1-4]. Technology is still being developed to get better image quality with lower energy consumption [5]. Recently, there has been introduced an idea of projecting images on a fog screen, [6-9] increasing the 3D effect even more. One of the natural applications for the technology is augmented reality, [10-12] which allows real and generated objects to be merged into one. The most user-friendly technology for the above application is a holographic near-eye display, due to its small dimensions, high energetic efficiency and 3D fidelity. Unfortunately, holographic imaging uses collimated beams, which usually results in small exit pupils [13] requiring e.g. eye tracking and other troublesome techniques. Other recurring problems are speckle noise and zero diffractive order, as it directs a portion of the incoming light into the eye of the user [9].

One of the possible solutions is the projection of computer-generated holograms (CGHs) on scatterings in liquids, along with the use of the fluorescence effect, e.g. in oils. The liquids would serve as volume screens capable of handling 3D virtual objects. The scatterings on particles of liquids would improve the projection by creating new light voxels dispersing the light around. Thus, the projection sustains its 3D and the exit pupil increases, as the beam spreads sideways. The scatterings observed are based on the Tyndall effect [14-15].

Passing through a liquid, the light emitted from the laser scatters on its particles (sized $40 \mathrm{~nm}$ to $900 \mathrm{~nm}$ - the same order of magnitude as the visible light). Due to these scatterings, which are propagating in all directions, the projected fields can be observed all around.

This is most advantageous for extending the exit pupil e.g. in near-eye displays. As a result, there is no necessity to have it accurately situated against the user's eye, as it is usually required for holographic displays.

Fluorescence occurs in different kinds of oils, [16-18] mainly due to chlorophyll and in tonic due to the presence of quinine. The principle of the phenomenon is as follows: the light beam with high energy causes excitation in molecules and emission of lower energy light. Due to the wavelength shift, with the use of a suitable filter, eliminating all the wavelengths except the excited ones, we can cut out the direct laser light and observe only the light coming from useful emission.

In this paper, we used olive oil, canola oil and engine oil as the media to project holographic images. With these oils a $532 \mathrm{~nm}$ laser was used as the exciting light source. Additionally, the experiment was repeated using tonic water, with a $405 \mathrm{~nm}$ laser. The fluids were stored at room temperature, tonic water was also kept without a cap, to eliminate any gas bubbles, which would negatively affect the projection. The liquids have been chosen to obtain a strong effect, while using common, non-toxic media.

Transmission spectra of test cells filled with different oils are presented in Fig. 1. In these figures, we can observe main peaks, corresponding with the light emitted from the laser source. Their wavelengths are $532 \mathrm{~nm}$ for the used oils and $405 \mathrm{~nm}$ for tonic water. In the part with longer wavelengths there are also visible smaller peaks, related to fluorescence.

In Fig. 1 transmission spectra of used color filters are presented. Their purpose was to separate the light coming from the excitation of fluid molecules, from the light emitted by the laser. For this reason, the filters were selected accordingly to transmit wavelengths corresponding to the fluorescence of a chosen liquid, and to block all the remaining spectrum.

The holograms were displayed using the setup presented in Fig. 2. The laser beam, after passing through a $\lambda / 2$ plate to correct polarization, is expanded into a 
converging beam with the use of a microscope lens positioned close to the focal spot of the positive lens.

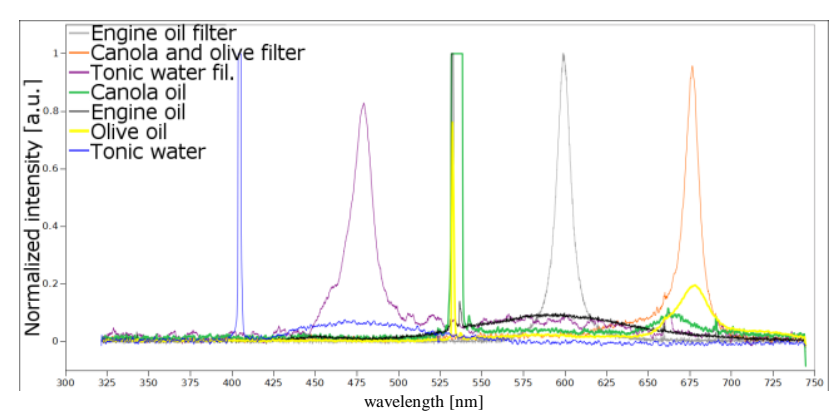

Fig. 1. Transmitted light spectra for used oils and adequate color filters.

The light passes through the beam splitter (BS), is reflected from the phase-only spatial light modulator (Holoeye Pluto), returns to BS and is directed towards a plastic, transparent cell containing oil. The distance between the container and the lens is set so that the hologram, observed in the beam waist, appears inside the container. Directly behind the container, there is an adequate color filter and then a camera which records the remaining light field. The distance from the SLM to the beam waist was $60 \mathrm{~mm}$, and from the waist to the camera $80 \mathrm{~mm}$.

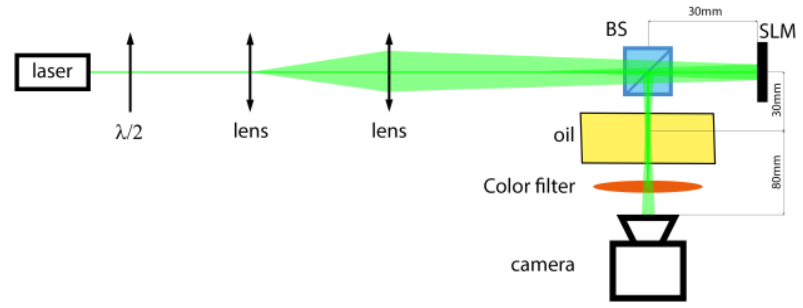

Fig. 2. Scheme of the experimental setup: BS - beam splitter, SLM spatial light modulator.

Holograms used in the projection were generated with in-house software executing the IFTA (Iterative Fourier Transform Algorithm) [19]. The CGHs contained planar circles and vertical lines, as presented in Fig. 3. To obtain the impression of full three dimensionality, the holograms were generated at different distances (the circle was located $10 \mathrm{~mm}$ closer to the camera) and displayed in a sequence on the SLM in time intervals of $100 \mathrm{~ms}$. As a result, the human eye perceived them as displayed simultaneously. The distances between them were short, so that both objects would appear inside the oil container.

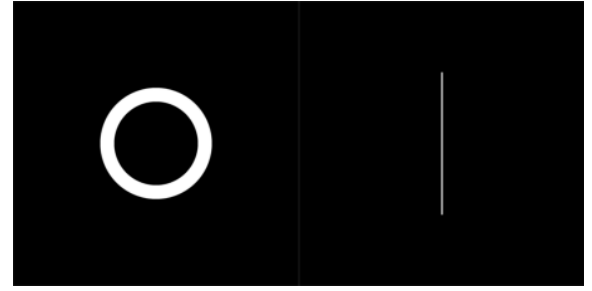

Fig. 3. Input images used to generate holograms: left: circle; right: line.

Figure 4 presents obtained images showing vertical lines and circles displayed alternately, as described before. Comparing images (a) and (b), we can see brighter images for the second one. This is related to stronger fluorescence of compounds contained in olive oil than in canola oil, as presented in Fig. 1. After using engine oil as a medium, we can see even better results, as shown in Fig. 4(c). The images displayed in this oil are brighter and exhibit higher contrast. This could be related to the fact that the composition of engine oil is more complicated, so the Tyndall effect will be stronger due to larger presence of very small particles. The projection can also be executed in tonic water, as presented in Fig. 5, which also shows a three-dimensional nature of displayed objects by focusing the camera at different distances.

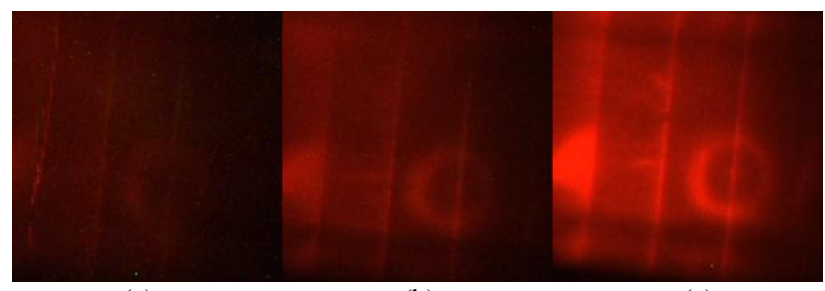

(a)

(b)

(c)

Fig. 4. Holographic projections captured with the same laser power and exposure parameters in (a) canola oil; (b) olive oil; (c) engine oil.

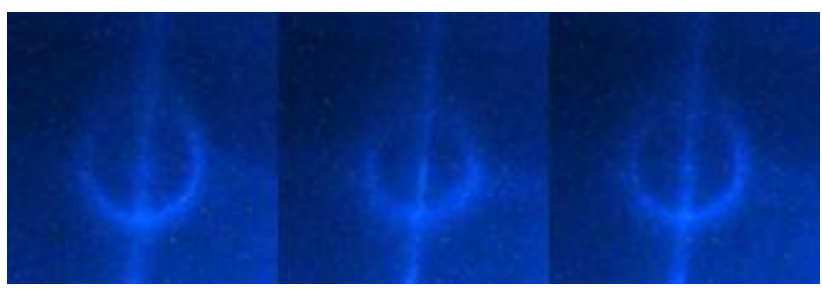

(a)

(b)

(c)

Fig. 5. Holograms displayed at different distances in tonic water. The camera is focused in plane of: (a) circle; (b) line; (c) in between.

To investigate the effect of using liquids as intermediate screens on the size of the exit pupil, the range of lateral shift of the camera was measured, while maintaining sharp images. The visibility of the phenomenon was the strongest for tonic water, so this liquid was used for measurements. Using the setup presented in Fig. 2, the camera was shifted in the direction perpendicular to the beam to the left and right, until the image observed started losing its sharpness and the shape 
of contours. Then the container and color filter were removed from the setup, and the measurements were repeated for bare laser illumination. Figure 6 presents images for boundary camera positions for tonic and Fig. 7 - after removing the container. With liquid, the maximum combined lateral shift was $8 \mathrm{~mm}$, while without liquid it was estimated at $6 \mathrm{~mm}$. The size of the image remained the same for both cases. Therefore, the exit pupil extension was observed to be c.a. $30 \%$ without introduction of any sophisticated optical constructions. Comparing Fig. 6 and Fig. 7, we can observe that, when using tonic, the output image is evenly bright, regardless of the camera location, which is very desirable in this kind of displays. Therefore, uniform performance of such display along the entire exit pupil was achieved.

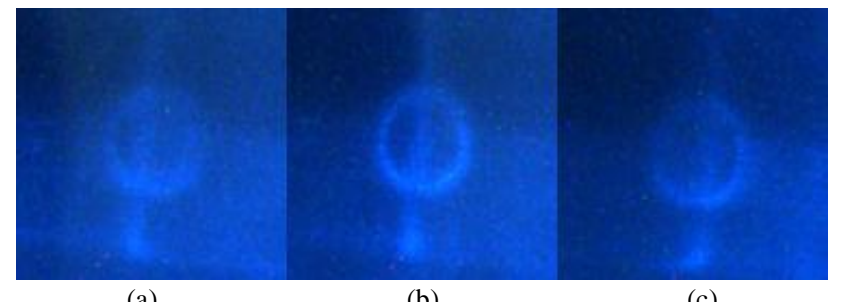

(a)

(b)

(c)

Fig. 6. Sideways shifting of the camera shows uniform image brightness and parallax of 3D images projected in the tonic water. Camera position: (a) $4 \mathrm{~mm}$ to the left; (b) on axis; (c) $4 \mathrm{~mm}$ to the right.

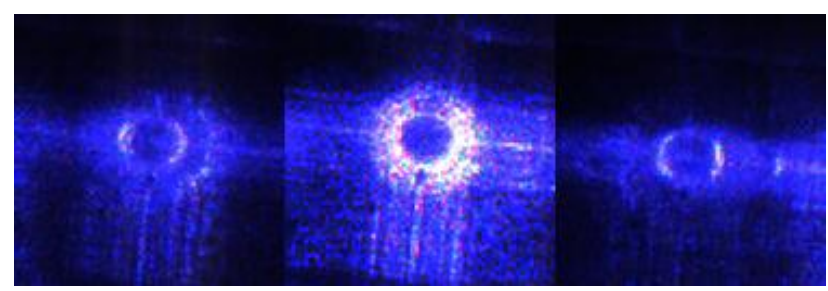

(a)

(b)

(c)

Fig. 7. Sideways shift of the camera without liquid with a $405 \mathrm{~nm}$ laser. Camera position: (a) $3 \mathrm{~mm}$ to the left; (b) on axis; (c) $3 \mathrm{~mm}$ to the right.

In order to further investigate the effect, additional measurement was performed. The beam-splitter and the SLM were taken out of the setup and the oil cell, color filter and camera were shifted to maintain initial distances (camera to beam waist $-80 \mathrm{~mm}$ ). The camera was then shifted sideways, while maintaining that distance, with $0.5^{\circ}$ steps, each time capturing an image.

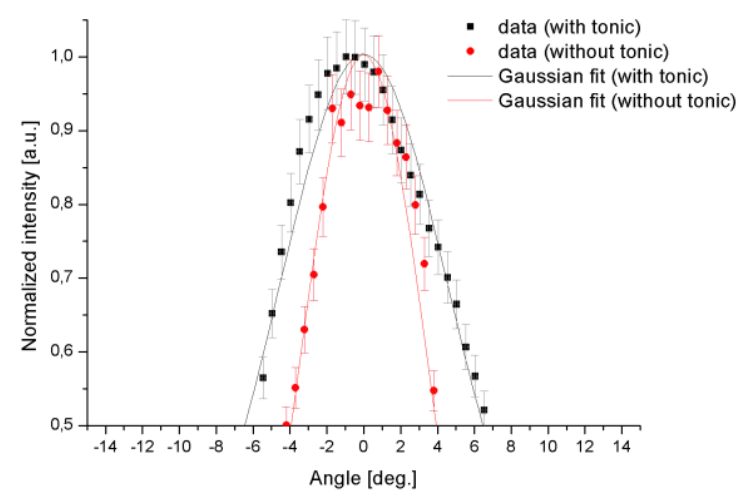

Fig. 8. The influence of tonic water on an angular size of the beam.

The integrals of images were calculated, to obtain intensities recorded for different angles, so that the beam divergence could be assessed. The results are presented in Fig. 8, indicating that the introduction of the liquid increased the angular size of the beam from c.a. $8^{\circ}$ to $13^{\circ}$. The characteristics is flatter, the image is more pleasant to the human eye and less dependent on the user's position.

In conclusions, we have demonstrated the method of realizing holographic projections on scattering centers in volumetric fluorescent liquids, and its possible application as intermediate screen for holographic near-eye displays. This setup could be potentially used to reduce the impact of the observer's position on image quality, stabilizing and improving the overall contrast and speckle noise of the displayed image, and additionally expanding the exit pupil, which is typically small in this kind of displays. Moreover, by careful selection of the fluorescent medium the proposed method allows holographic projections in arbitrary colors, independent of the wavelength of the excitation laser.

This work was supported by the „HANEDA” project carried out under contract no. POIR.04.04.00- 003DD9/16-00 within the TEAM-TECH program of the Foundation for Polish Science, co-financed by the European Union under the European Regional Development Fund.

\section{References}

[1] X. Li, C.P. Chen, H. Gao et al., J. Display Technol. 10(6), 438 (2014)

[2] X. Li, Z. Song, F. Li, X. Dong, W. Liu, SID Symposium Digest of Technical Papers 48(1), 344 (2017). doi: 10.1002/sdtp.11850

[3] T. Sasaki et al., Appl. Phys. Lett. 102, 063306 (2013). doi: 10.1063/1.4792735

[4] N. Tsutsumi, K. Kinashi, A. Nomura, W. Sasaki, Materials 5(8), 1477 (2012). doi: 10.3390/ma5081477

[5] M. Makowski et al., Opt. Express 20(22), 25130 (2012).

[6] A. Yagi, M. Imura, Y, Kuroda, O. Oshiro, SIGGRAPH Asia 2011 Emerging Technologies, doi: 10.1145/2073370.2073388 
[7] C.H. Hsu, K.L. Hua, W.H. Cheng. SIGGRAPH Asia 2012 Posters, doi: 10.1145/2407156.2407189

[8] Z. Zeng, H. Zheng, X. Lu, H. Gao, Y. Yu, Opt. Rev. 22, 853 (2015). https://doi.org/10.1007/s10043-015-0109-2

[9] I. Rakkolainen, 3DTV Conference, Tampere 2010, doi: 10.1109/3DTV.2010.5506314

[10] S. Yanfeng et al., Optik 157, 190 (2018).

[11] G. Li, D. Lee, Y. Jeong, J. Cho, B. Lee, Opt. Lett. 41(11), 2486 (2016).

[12] C.L. Lin, Y.Z. Su, M.W. Hung, K.C. Huang, Proc. SPIE 7798 , 779826 (2010).

[13] A. Maimone, A. Georgiou, J.S. Kollin, ACM Trans. Graph. 36(4), 1 (2017). doi: 10.1145/3072959.3073624

[14] M. Quinten, Optical properties of nanoparticle systems: Mie and beyond (John Wiley \& Sons 2010).

[15] J.-W. Liaw, S.-W. Tsai, H.-H. Lin, T.-C. Yen, B.-R. Chen, J. Quantitative Spectroscopy and Radiative Transfer 113(17), 2234 (2012). doi: 10.1016/j.jqsrt.2012.08.002

[16] T. Mu et al., Anal. Methods 5, 6960 (2013). doi: 10.1039/c3ay40987b

[17] T. Mu et al., Analytical Lett. 49(8), 1233 (2015). doi: 10.1080/00032719.2015.1086777

[18] V. Rostampour, M.J. Lynch, WIT Transactions on Ecology and the Environment 95, 265262 (2006). doi: 10.2495/WP060261

[19] F. Wyrowski, O. Bryngdahl, Opt. Soc. Am. A 5(7), 1058 (1988). doi: 10.1364/JOSAA.5.001058 